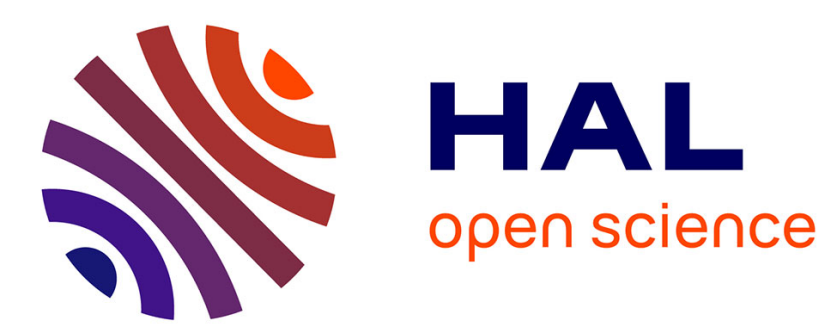

\title{
Endoarterial treatment in combination with percutaneous thermoablation for medium-sized and oligonodular hepatocellular carcinomas \\ Dann Ouizeman
}

\section{- To cite this version:}

Dann Ouizeman. Endoarterial treatment in combination with percutaneous thermoablation for medium-sized and oligonodular hepatocellular carcinomas. Human health and pathology. 2019. dumas-02435472

\section{HAL Id: dumas-02435472 https://dumas.ccsd.cnrs.fr/dumas-02435472}

Submitted on 10 Jan 2020

HAL is a multi-disciplinary open access archive for the deposit and dissemination of scientific research documents, whether they are published or not. The documents may come from teaching and research institutions in France or abroad, or from public or private research centers.
L'archive ouverte pluridisciplinaire $\mathbf{H A L}$, est destinée au dépôt et à la diffusion de documents scientifiques de niveau recherche, publiés ou non, émanant des établissements d'enseignement et de recherche français ou étrangers, des laboratoires publics ou privés. 
Université de Nice Sophia Antipolis

Faculté de Médecine
Université

nice

Sophia Antipolis

\section{TRAITEMENTS ENDOARTERIEL ET PERCUTANE COMBINES EN UN TEMPS \\ DANS LE TRAITEMENT DU CARCINOME HEPATOCELLULAIRE DANS LES \\ CRITERES DE MILAN}

Thèse d'exercice de Médecine

(Diplôme d'Etat)

Présentée et soutenue publiquement le 11 Septembre 2019 à Nice

Par

Dann Joseph Anthony OUIZEMAN

Né le 14 Novembre 1990 à Paris XXe arrondissement

Discipline : Hépato-Gastro-Entérologie

Composition du jury de thèse :

Monsieur le Professeur Thierry PICHE — - Président du jury

Monsieur le Professeur Rodolphe ANTY - Directeur de thèse

Monsieur le Docteur Emmanuel CHAMOREY - Assesseur

Monsieur le Professeur Patrick CHEVALLIER - Assesseur

Monsieur le Professeur Xavier HEBUTERNE - Assesseur 
Université

nice

Sophia Antipolis

Faculté de Médecine

UNIVERSITE DE NICE SOPHIA ANTIPOLIS

FACULTE DE MEDECINE

Liste des enseignants au 1er septembre 2018 à la Faculté de Médecine de Nice

\section{Doyen}

Pr. BAQUÉ Patrick

Vice-doyens

$\begin{array}{ll}\text { Pédagogie } & \text { Pr. ALUNNI Véronique } \\ \text { Recherche } & \text { Pr DELLAMONICA Jean } \\ \text { Etudiants } & \text { M. JOUAN Robin } \\ \text { projet Campus } & \text { Pr. PAQUIS Philippe }\end{array}$

Chargé de mission projet Campus Pr. PAQUIS Philippe

Conservateur de la bibliothèque Mme AMSELLE Danièle

Directrice administrative des services Mme CALLEA Isabelle

Doyens Honoraires M. RAMPAL Patrick

M. BENCHIMOL Daniel 
M. AMIEL Jean

M. BAQUÉ Patrick

M. BERNARDIN Gilles

M. BOILEAU Pascal

M. DARCOURT Jacques

M. ESNAULT Vincent

M. FENICHEL Patrick

M. FUZIBET Jean-Gabriel

M. GILSON Éric

M. GUGENHEIM Jean

M. HASSEN KHODJA Reda

M. HÉBUTERNE Xavier

M. HOFMAN Paul

Mme ICHAI Carole

M. LACOUR Jean-Philippe

M. LEFTHERIOTIS Geogres

M. MARQUETTE Charles-Hugo

M. MARTY Pierre

M. MICHIELS Jean-François

M. MOUROUX Jérôme

Mme PAQUIS Véronique

M. PAQUIS Philippe

M. QUATREHOMME Gérald

M. RAUCOULES-AIMÉ Marc

M. ROBERT Philippe

M. SANTINI Joseph

M. THYSS Antoine

M. TRAN Albert
Urologie (52.04)

Anatomie - Chirurgie Générale (42.01)

Réanimation Médicale (48.02)

Chirurgie Orthopédique et Traumatologique (50.02)

Biophysique et Médecine Nucléaire (43.01)

Néphrologie (52-03)

Biologie du Développement et de la Reproduction (54.05)

Médecine Interne (53.01)

Biologie Cellulaire (44.03)

Chirurgie Digestive (52.02)

Chirurgie Vasculaire (51.04)

Nutrition (44.04)

Anatomie et Cytologie Pathologiques (42.03)

Anesthésiologie et Réanimation Chirurgicale (48.01)

Dermato-Vénéréologie (50.03)

Chirurgie vasculaire; médecine vasculaire (51.04)

Pneumologie (51.01)

Parasitologie et Mycologie (45.02)

Anatomie et Cytologie Pathologiques (42.03)

Chirurgie Thoracique et Cardiovasculaire (51.03)

Génétique (47.04)

Neurochirurgie (49.02)

Médecine Légale et Droit de la Santé (46.03)

Anesthésie et Réanimation Chirurgicale (48.01)

Psychiatrie d'Adultes (49.03)

O.R.L. (55.01)

Cancérologie, Radiothérapie (47.02)

Hépato Gastro-entérologie (52.01) 
Mme ASKENAZY-GITTARD Florence

M. BARRANGER Emmanuel

M. BÉRARD Étienne

Mme BLANC-PEDEUTOUR Florence

M. BONGAIN André

Mme BREUIL Véronique

M. CASTILLO Laurent

M. CHEVALLIER Patrick

M. DE PERETTI Fernand

M. DRICI Milou-Daniel

M. FERRARI Émile

M. FERRERO Jean-Marc

M. FONTAINE Denys

M. GIBELIN Pierre

M. HANNOUN-LEVI Jean-Michel

M. LEVRAUT Jacques

M. LONJON Michel

M. MOUNIER Nicolas

M. PADOVANI Bernard

M. PICHE Thierry

M. PRADIER Christian

Mme RAYNAUD Dominique

M. ROSENTHAL Éric

M. SCHNEIDER Stéphane

M. STACCINI Pascal

M. THOMAS Pierre

M. TROJANI Christophe
Pédopsychiatrie (49.04)

Gynécologie Obstétrique (54.03)

Pédiatrie (54.01)

Cancérologie - Génétique (47.02)

Gynécologie-Obstétrique (54.03)

Rhumatologie (50.01)

O.R.L. (55.01)

Radiologie et Imagerie Médicale (43.02)

Anatomie-Chirurgie Orthopédique (42.01)

Pharmacologie Clinique (48.03)

Cardiologie (51.02)

Cancérologie ; Radiothérapie (47.02)

Neurochirurgie (49.02)

Cardiologie (51.02)

Cancérologie ; Radiothérapie (47.02)

Médecine d'urgence (48.05)

Neurochirurgie (49.02)

Cancérologie, Radiothérapie (47.02)

Radiologie et Imagerie Médicale (43.02)

Gastro-entérologie (52.01)

Épidémiologie, Économie de la Santé et Prévention (46.01)

Hématologie (47.01)

Médecine Interne (53.01)

Nutrition (44.04)

Biostatistiques et Informatique Médicale (46.04)

Neurologie (49.01)

Chirurgie Orthopédique et Traumatologique (50.02) 
Mme ALUNNI Véronique

M. ANTY Rodolphe

M. BAHADORAN Philippe

Mme BAILLIF Stéphanie

Mme BANNWARTH Sylvie

M. BENIZRI Emmanuel

M. BENOIT Michel

M. BOZEC Alexandre

M. BREAUD Jean

M. CHEVALIER Nicolas

Mme CHINETTI Giulia

M. CLUZEAU Thomas

M. DELLAMONICA Jean

M. DELOTTE Jérôme

M. FOURNIER Jean-Paul

Mlle GIORDANENGO Valérie

Mme GIOVANNINI-CHAMI Lisa

M. GUÉRIN Olivier

M. IANNELLI Antonio

M. ILIE Marius

M JEAN BAPTISTE Elixène

M. PASSERON Thierry

M. ROGER Pierre-Marie

M. ROHRLICH Pierre

M. ROUX Christian

M. RUIMY Raymond

Mme SACCONI Sabrina

M. SADOUL Jean-Louis
Médecine Légale et Droit de la Santé (46.03)

Gastro-entérologie (52.01)

Cytologie et Histologie (42.02)

Ophtalmologie (55.02)

Génétique (47.04)

Chirurgie Générale (53.02)

Psychiatrie (49.03)

ORL- Cancérologie (47.02)

Chirurgie Infantile (54-02)

Endocrinologie, Diabète et Maladies Métaboliques (54.04)

Biochimie-Biologie Moléculaire (44.01)

Hématologie (47.01)

réanimation médicale (48.02)

Gynécologie-obstétrique (54.03)

Thérapeutique (48-04)

Bactériologie-Virologie (45.01)

Pédiatrie (54.01)

Méd. In ; Gériatrie (53.01)

Chirurgie Digestive (52.02)

Anatomie et Cytologie pathologiques (42.03)

Chirurgie vasculaire (51.04)

Dermato-Vénéréologie (50-03)

Maladies Infectieuses; Maladies Tropicales (45.03)

Pédiatrie (54.01)

rhumatologie (50.01)

Bactériologie-virologie (45.01)

Neurologie (49.01)

Endocrinologie, Diabète et Maladies Métaboliques (54.04) 
M. AMBROSETTI Damien

M. BENOLIEL José

Mme BERNARD-POMIER Ghislaine

M. BRONSARD Nicolas

Mme BUREL-VANDENBOS Fanny

M. DOGLIO Alain

M DOYEN Jérôme

M FAVRE Guillaume

M. FOSSE Thierry

M. GARRAFFO Rodolphe

Mme HINAULT Charlotte

M. HUMBERT Olivier

Mme LAMY Brigitte

Mme LONG-MIRA Elodie

Mme MAGNIÉ Marie-Noëlle

Mme MOCERI Pamela

M. MONTAUDIE Henri

Mme MUSSO-LASSALLE Sandra

M. NAÏMI Mourad

Mme POMARES Christelle

M. SAVOLDELLI Charles

Mme SEITZ-POLSKI barbara

M. TESTA Jean

M. TOULON Pierre
Cytologie et Histologie (42.02)

Biophysique et Médecine Nucléaire (43.01)

Immunologie (47.03)

Anatomie Chirurgie Orthopédique et Traumatologique (42.01)

Anatomie et Cytologie pathologiques (42.03)

Bactériologie-Virologie (45.01)

Radiothérapie (47.02)

Néphrologie (52.03)

Bactériologie-Virologie-Hygiène (45.01)

Pharmacologie Fondamentale (48.03)

Biochimie et biologie moléculaire (44.01)

Biophysique et Médecine Nucléaire (43.01)

Bactérilogie-virologie (45.01)

Cytologie et Histologie (42.02)

Physiologie (44.02)

Cardiologie (51.02)

Dermatologie (50.03)

Anatomie et Cytologie pathologiques (42.03)

Biochimie et Biologie moléculaire (44.01)

Parasitologie et mycologie (45.02)

Chirurgie maxillo-faciale et stomatologie (55.03)

Immunologie (47.03)

Épidémiologie Économie de la Santé et Prévention (46.01)

Hématologie et Transfusion (47.01) 
PROFESSEUR DES UNIVERSITÉS

M. HOFLIGER Philippe Médecine Générale (53.03)

MAITRE DE CONFÉRENCES DES UNIVERSITÉS

M. DARMONDavid Médecine Générale (53.03)

PROFESSEURS AGRÉGÉS

Mme LANDI Rebecca Anglais

PRATICIEN HOSPITALIER UNIVERSITAIRE

M. DURAND Matthieu Urologie (52.04)

PROFESSEURS ASSOCIÉS

M. GARDON Gilles Médecine Générale (53.03)

Mme MONNIER Brigitte Médecine Générale (53.03)

MAITRES DE CONFÉRENCES ASSOCIÉS

Mme CASTA Céline Médecine Générale (53.03)

M. GASPERINI Fabrice Médecine Générale (53.03)

M. HOGU Nicolas Médecine Générale (53.03) 
Constitution du jury en qualité de 4ème membre

\section{Professeurs Honoraires}

M ALBERTINI Marc

M. BALAS Daniel

M. BATT Michel

M. BLAIVE Bruno

M. BOQUET Patrice

M. BOURGEON André

M. BOUTTÉ Patrick

M. BRUNETON Jean-Noël

Mme BUSSIERE Françoise

M. CAMOUS Jean-Pierre

M. CANIVET Bertrand

M. CASSUTO Jill-patrice

M. CHATEL Marcel

M. COUSSEMENT Alain

Mme CRENESSE Dominique

M. DARCOURT Guy

M. DELLAMONICA Pierre

M. DELMONT Jean

M. DEMARD François

M. DESNUELLE Claude

M. DOLISI Claude

Mme EULLER-ZIEGLER Liana

M. FRANCO Alain

M. FREYCHET Pierre
M. GASTAUD Pierre

M. GÉRARD Jean-Pierre

M. GILLET Jean-Yves

M. GRELLIER Patrick

M. GRIMAUD Dominique

M. JOURDAN Jacques

M. LAMBERT Jean-Claude

M. LAZDUNSKI Michel

M. LEFEBVRE Jean-Claude

M. LE FICHOUXYves

Mme LEBRETON Elisabeth

M. MARIANI Roger

M. MASSEYEFF René

M. MATTEI Mathieu

M. MOUIEL Jean

Mme MYQUEL Martine

M. ORTONNE Jean-Paul

M. PRINGUEY Dominique

M. SAUTRON Jean Baptiste

M. SCHNEIDER Maurice

M. TOUBOL Jacques

M. TRAN Dinh Khiem

M VAN OBBERGHEN Emmanuel

M. ZIEGLER Gérard 
M.C.U. Honoraires

M. ARNOLD Jacques

M. GIUDICELLI Jean

M. BASTERIS Bernard

M. MAGNÉ Jacques

Mlle CHICHMANIAN Rose-Marie

Mme MEMRAN Nadine

Mme DONZEAU Michèle

M. MENGUAL Raymond

M. EMILIOZZI Roméo

M. PHILIP Patrick

M. FRANKEN Philippe

M. POIRÉE Jean-Claude

M. GASTAUD Marcel

Mme ROURE Marie-Claire 


\section{REMERCIEMENTS}

\section{Aux Membres du Jury,}

\section{A monsieur le Professeur Thierry PICHE,}

Vous me faites l'honneur de présider mon jury de thèse et de juger mon travail. Merci d'avoir cru en moi dès notre première rencontre. Au-delà de la médecine, vous m'avez coaché pendant les quatre années d'internat et avez éveillé mon intérêt pour la recherche clinique. Merci de m'avoir poussé à atteindre mes objectifs. Soyez assuré de ma profonde gratitude.

\section{A monsieur le Docteur Emmanuel CHAMOREY,}

Merci de me faire l'honneur de siéger dans mon jury de thèse. Merci pour l'implication de votre équipe qui a participé à l'aboutissement de ce travail, en espérant qu'il ne soit que le premier d'une solide collaboration. Avec tout mon respect.

\section{A monsieur le Professeur Patrick CHEVALLIER,}

Merci de me faire l'honneur de siéger dans mon jury de thèse. Merci de toujours rester disponible pour nous donner conseils et explications, pour les relectures d'examens, et ce même à minuit, un soir de réveillon. Merci de votre implication auprès des patients. Soyez assuré de mon plus grand respect.

\section{A monsieur le Professeur Xavier HEBUTERNE,}

Merci de me faire l'honneur de siéger dans mon jury de thèse. Vous êtes à mes yeux un exemple de médecin qui est resté proche des patients malgré les compétences et le statut. J'admire votre façon de vous asseoir au lit des patients pour prendre le temps nécessaire. Merci de prendre le temps également avec tous ceux qui sont disposés à apprendre (étudiants, médecins et mêmes patients). Votre empathie force le respect et est un modèle pour moi. Je vous remercie pour tout ce que vous m'avez enseigné.

\section{A monsieur le Professeur Rodolphe ANTY,}

Merci de m'avoir fait l'honneur de diriger mon travail de thèse. Merci de m'avoir pris sous ton aile et de continuer à me transmettre chaque jour tes innombrables compétences cliniques (de l'examen endobuccal au TR). Merci de t'être positionné en tant que maître en médecine mais également d'endosser le rôle de grand frère quand j'en ai besoin. En plus du savoir médical, tu m'as enseigné la grandeur d'esprit. Ton dévouement fait de toi un modèle. Je te remercie pour ton soutien indéfectible et ta disponibilité. Merci d'avoir été à mes côtés ces quatre dernières années et d'avoir respecté mes volontés. Merci pour les longues heures de discussion parfois à des horaires pas possibles. Je ne trouve pas les mots pour exprimer tout ce que je te dois. Merci infiniment. 


\section{A mes Aînés,}

\section{A monsieur le Professeur Albert TRAN,}

Merci de nous pousser dans nos retranchements afin de nous mener toujours plus haut. Merci de nous enseigner l'esprit de synthèse et de précision. J'admire votre façon de gérer les équipes avec pragmatisme, tel un entrepreneur. Merci de m'avoir accueilli dans le service avec bienveillance et de continuer à me transmettre vos compétences en Hépatologie.

\section{A monsieur le Professeur Stéphane SCHNEIDER,}

Je n'ai pas eu la chance de faire un semestre complet à l'USN, cependant tu m'as énormément appris. Merci d'avoir réussi à débloquer ce fameux $3^{\mathrm{e}}$ poste d'interne qui m'a permis d'accéder à la si belle ville de Nice. En plus d'être un maître en nutrition, tu as des connaissances sur de nombreux sujets, que tu sais nous enseigner avec une pointe d'humour toujours bien dosée. Sois assuré de tout mon respect.

\section{A monsieur le Professeur Geoffroy VANBIERVLIET,}

Merci pour le temps passé ensemble en endoscopie. Merci pour ta droiture qui nous pousse à toujours tirer le meilleur de nous-même, tout en nous faisant confiance. Merci pour ton implication dans l'enseignement et milles mercis de m'avoir permis de participer aux universités d'endoscopie. Sois assuré de toute ma reconnaissance.

\section{A monsieur le Professeur Robert BENAMOUZIG,}

Merci pour votre gentillesse. Merci d'avoir pris le temps à plusieurs reprises de m'aiguiller dans les choix qui m'ont finalement mené où je suis aujourd'hui. Soyez assuré de toute ma gratitude.

\section{A monsieur le Professeur Frédéric ADNET,}

Merci de m'avoir accueilli dans votre service où j'ai tant appris.

\section{A monsieur le Professeur Jean-Claude TRINCHET,}

Merci de m'avoir enseigné mes premières notions d'Hépatologie. Votre souvenir restera un monument dans la discipline.

A Jérôme FILIPPI et Ophélie ANTUNES, de m'avoir guidé dans mes premiers pas d'interne de gastroentérologie et en endoscopie.

A Anne-Claire FRIN et Delphine OUVRIER, de m'avoir fait fumer passivement tous les matins avant la visite. Plus sérieusement, merci de votre bonne humeur qui a rendu agréable mon stage en cancérologie.

A Marie-Lise MONTOYA, ma maman du CHU. Je t'ai appréciée au-delà de notre relation professionnelle. Tu es une personne exceptionnellement humaine, et les patients le ressentent (les seuls que j'ai vus attendre 3 mois de délai avant la consultation juste pour te dire merci !). Merci pour tes petites attentions au retour de voyage. J'espère que ta retraite dans les îles sera aussi belle que ta carrière. 
A Kamel ARAB, Kamel, Kamel, Kamel... Tu m'as toujours fait confiance et m'as énormément appris (que ce soit GPE, endoscopie ou des blagues un peu douteuses). Tu m'as permis de passer des heures en salle d'endoscopie et toujours dans la bonne humeur. Ta gentillesse est légendaire et digne du respect de tous. Merci pour tous tes conseils sur la vie en général. Tu es pour moi bien plus qu'un chef.

A Eve GELSI et Cécile GOMERCIC, j'ai énormément apprécié mon passage aux SMC. Eve, merci pour ta rigueur, ta franchise et pour les urgences endoscopiques toujours improbables que l'on rencontre quand tu es d'astreinte. Cécile, merci de ta patience qui permet à chaque interne d'apprendre à poser une voie centrale (même si je ne suis pas ta plus grande réussite...). Et merci pour tes qualités humaines. Merci à vous deux de nous faire partager vos compétences cliniques et endoscopiques.

A Régine TRUCHI, pour ta gentillesse et ton engagement auprès des patients. Tu es un exemple de volonté et tu parviens à faire de grandes choses par ton implication, dans toutes les unités où tu exerces. Je suis heureux que l'on puisse travailler ensemble davantage.

A Julie BENARD-LAME, pour ta sympathie et ton efficacité dans le service.

A Nadia ARAB, de répondre à toutes mes questions sur les $\mathrm{MICl}$ et les protocoles. Tu cherches toujours à aider les patients peu importe les problèmes, ton empathie est exemplaire.

A Franck SOUSSI, merci de m'avoir reçu en consultation à plusieurs reprises et de m'avoir fait partager tes connaissances et ta bonne humeur.

A Daniel BIANCHI, merci d'être aussi proche des internes et de toujours nous soutenir à fond (notamment au GastroQuizz !). Merci de nous faire confiance en endoscopie et de prendre le temps de discuter avec chacun de nous.

A Laurianne DE GALLEANI, de m'avoir accueilli dans ton bloc pour m'apprendre l'utilisation des traitements instrumentaux en proctologie.

A Dov BELHASSEN, de m'avoir permis d'assister à mes premières endoscopies et de m'avoir aiguillé sur la spécialité que j'aime tant aujourd'hui.

A Eva AFFLALO, je devrais dire "Docteur Afflalo". Plus qu'un pédiatre ou un médecin traitant, vous avez été un exemple pour moi. Je vous idéalise depuis toujours. Votre relation aux patients est la raison qui m'a donné envie de faire médecine. Votre façon de pratiquer la médecine donne un sens à chaque mot du serment d'Hippocrate. Votre contribution dans ma vie est majeure.

A Chrystelle TREZIERE, mes quelques mois de stage avec vous m'ont fait découvrir une médecine générale de qualité.

A l'équipe de Médecine Nucléaire de l'Archet: Micheline RAZZOUK, Philippe VIAU, Guillaume NIVAGGIONI, de m'avoir accueilli et présenté votre spécialité (les scanners avec les couleurs...).

A l'équipe des Urgences d'Avicenne : Birol, Charlotte, Hayatte, Lilia, Louis-Etienne, Lydia, Maguy, Paul-Georges, Pranesh, Roxana, Sabine, Sheila, et les autres ... 


\section{A mes co-internes,}

A Clémence CANIVET, d'avoir été mon exemple en tant qu'interne. J'espère pouvoir assurer autant que tu ne l'as fait dans le service. Merci pour tout ce que tu m'as appris.

A Léa LOMBARDI, merci pour tous tes conseils bébé.

A Thibaut DELASALLE, notre semestre ensemble en cancérologie a été une incroyable rencontre humaine. J'espère que notre route se recroisera rapidement.

A Grégoire BESSI, d'avoir pris le temps de me présenter le service quand j'étais en stage à l'Archet 1.

A Audrey HASTIER-DE CHELLES, pour ta gentillesse, ta solidarité et ta force d'organisation qui font de toi un excellent médecin et une personne formidable.

A Clément FORTIER-BEAULIEU, pour ton sérieux et tes conseils avisés en endoscopie.

A Edouard LARREY et Maude DESCHAMPS DE BOISHEBERT, j'ai adoré faire équipe avec vous. Entre les crises existentielles de Maude et la zen-attitude d'Edouard, on a quand même passé quatre belles années ensemble. J'espère qu'on ne se perdra pas de vu.

A Juliette LAVEISSIERE, avec qui j'ai été le plus souvent binôme. Ça a été un plaisir. Ton dynamisme et ta force de caractère sont capables de te mener très loin.

A Adrien NICOLAU, qui est toujours de bonne humeur. Tu apportes une stabilité à l'équipe tout en envoyant des punchlines assassines.

A Morgane SALLETTE, Léa MARIE, et Marie DE MATHAREL, bon courage pour la suite de votre internat dans notre belle spécialité.

A mes co-internes non gastro : Marion (the first-one), Théo, Margaux, Angélique, Perrine, Wulfran, Imane et Al-Aydin.

A mes co-internes des urgences d'Avicenne: Sujeewa et Yasmine (quel trio mémorable !!!), Sylvie, Morgane, Cecilia et Rhyzlene.

\section{Aux autres spécialités,}

Aux Anesthésistes Réanimateurs : Rémi, Mathilde, Romain, Olivier, Andrea, et tous les autres, de nous sauver chaque nuit quand ça part en vrille dans les services et d'avoir la patience de nous assister en endoscopie.

Aux Chirurgiens : Pr IANNELLI pour ta confiance et ta bonne humeur, Pr GUGENHEIM, $\operatorname{Pr}$ BENIZRI, Dr RAHILI, Manuela, Alexandre, Clémentine, Lionel, Benjamin, et tous les autres.

A Magalie HAMM-ORLOWSKI et Dr CHERIKH, pour votre gentillesse et votre patience.

Aux radiologues : Julien, Madleen, Fabrice et $\mathrm{Dr}$ TRAN, pour le temps que vous nous accordez. 


\section{Aux équipes soignantes,}

Du $4^{\circ} \mathrm{B}$ : Alexia, Arielle, Asma, Aude, Carole, Céline, Franck, Julie, Maeva, Paolo, Rémi, Roukia, Stéphanie, ... ceux et celles qui me connaissent depuis le plus longtemps.

D’Hépatologie : Alain, Anaïs, Audrey, Cécile et ses tortues, Gourette, Juliette, Laurence, Madely, Magalie, Marie, Marilyne, Martine, Nadège, Olivier, Ridha, Sabino, Sandrine, Vanessa, Véronique $G$, Véronique $L$ (la faucheuse), ..., pour l'équipe formidable que vous formez, toujours motivée pour améliorer les choses.

A Marie-Pierre GILLY, pour ton travail et ton implication extraordinaire.

De Cancérologie : Aurélie, Blandine, Claire, Eva, Gelissa, Ingrid, Jenny, Jocelyne, Johanna, Laurence, Lorène, Marylou, Maud, Nolwenn, Romane, Safia, Sandrine, Sandy, Sophie, Tiffany ... d'avoir réalisé mon dernier jour de stage le plus mémorable.

De I'HDS : Audrey, Brigeou, Cyrielle, Isabelle, Laure, Lucilia, Marie, Marlène, Sylvie, pour l'incroyable bonne humeur qui règne dans le service.

De l'EFD : l'infatigable Paupau et Marie-Hélène.

Du SMC : Carine, Céline, Claude, Elisabeth et ses éventails designs, Éric, Fafa, Floriane, Juju, Jocelyne qui est bien plus jeune que mon père, Laurent, Océ, Olivier, Sarah, Stéph, Valentina, Valérie, Véro, Séverine, ... seulement 4 mois de stage mais des relations fortes créés avec chacun d'entre vous.

De I'USN : Christine, Claire, Eva, Jessica, Najouah, Nathalie pour ton implication exemplaire, Laura, Nadège, Rabeb, Sab, ...

De I'Endoscopie: Alexandra, Bernard, Emilie, Elodie, Franck, Jean-Pierre, Georgiana, Gérard, Jean-Yves, Marc, Marion, Mathilde, Micka, Stefania,...

De I'HDJ : Alicia, Christophe, Éric, Isabelle, Jean, Marie-Pierre, Noëlle, Siam ...

Du Pôle : Emmanuelle, Elise, Céline, ... pour le travail formidable que vous effectuez.

Du CSAPA : Cécile et Nathalie pour l'ambiance, les compétences et l'implication.

De I’URC : Aaron, Hélène, Jessica, Joris, Virginie

Des services de chirurgie

\section{De Médecine Nucléaire et des Urgences d'Avicenne}

Aux secrétaires, Annie, Brigitte, Céline, Christine, Chrystelle, Colette, Corinne, Diane, Dominique, Florence, Graziella, Laetitia, Megan, Roselyne, Sandrine, Sylvie, Virginie, ... d'avoir passé de longues heures à écouter ma douce voix qui ne parle pas du tout trop vite.

Aux diététiciennes, Delphine, Evelyne, Isabelle, Katia et Mégane.

Aux assistantes sociales, Johanna et Marina.

Aux cadres infirmiers, Chrystelle, Elodie, Eya, Jean-Jacques, Khanika, Magalie, Marcelle, Nathalie, Séverine, Victor, mais aussi aux cadres secrétaires Mme Liberatore et Mme Noble.

Un remerciement tout particulier aux équipes de nuit... 


\section{A ma famille,}

A mes parents Patrick et Sonia, de m'avoir transmis les bases qui m'ont permis d'évoluer dans la vie. Merci d'avoir cru en moi et de m'avoir transmis la valeur de la famille et de l'effort. Papa, tu es mon exemple de courage et de persévérance. Maman, tu m'as soutenu, poussée et même tenu par la main pendant ma scolarité (et même pendant la p1). Je vous aime.

A David, pour tous les bons moments partagés pendant notre enfance. Merci à Julia et toi pour mon neveu à venir.

A Keren, pour tes petits plats attentionnés et tes gratouilles dans le dos. Je te souhaite une carrière pleine d'épanouissement.

A Dov, mon bébé qui est maintenant un homme, chaque moment passé avec toi me remplit de bonheur. Tu me manques énormément. Je suis tellement fier de toi.

A Samuel, mon cousin/frère farfelu, merci d'être toi et d'égayer ma vie. Merci pour tes colorations capillaires incroyables et tout le reste.

A mes grands-parents Paul et Jacqueline, pour votre soutien inconditionnel et votre présence permanente. Merci pour tous les moments passés ensemble.

A mes grands-parents David et Margotte, pour l'amour que vous m'avez transmis et d'avoir grandement contribué à mon éducation.

A Franck, pour ta bonne humeur, tes blagues incroyables et tes multiples attentions. A Carole, de m'avoir considéré comme un fils.

A Jacky, mon deuxième père, tu as toujours été présent dans les bons et les mauvais moments. Tu me manques au quotidien. Merci de nous avoir amené Mélisa qui a exactement les mêmes qualités que toi.

A Didier et Schelly, pour votre gentillesse et votre générosité.

A Avi et Corinne, pour nos discussions interminables. Merci d'être présent et de nous faire partager de super moments. Au-delà des mathématiques, je vous dois une bonne partie de ma culture générale.

A Franck et Linda, d'être toujours là pour nous.

A Jennifer, Mickael, Kevin, Samuel, Kevin, Sacha, Ilona, Julien, Shana, Gabou, Nathan, Nina, David, Shanaelle, Léane et Lola

A ceux qui nous ont quittés et qui nous manquent tant.

A mes beaux-parents Jean-Jacques et Nicole, d'être aussi extraordinaires, on ne pourrait rêver meilleurs beaux-parents. Votre abnégation force l'exemple. Merci pour votre soutien permanent. Soyez assurés de tout mon respect et ma gratitude.

A la famille Bensimon, et mes plus vieux neveux pour ces merveilleux moments passés au Canada. 
A Michael, mon frère d'adoption. Merci pour ton aide constante, tes conseils avisés et nos conversations nocturnes du vendredi soir jusqu'à pas d'heures.

A madame le Docteur Audrey Sultan, soyez assuré de tout mon respect.

A Maxime et Audrey, de nous recevoir à chaque voyage à Paris. Merci pour toutes nos rigolades et toutes les pizzas partagées. A mes neveux Lok d'amour.

A tata Clémence et tonton Daniel (tu ne pourras pas y échapper), de nous avoir reçus chez vous sans oublier que vous étiez chez nous. Merci pour tout et merci encore d'embellir notre vie niçoise. Merci aussi à Amiel, Daphné, Youval, Batsheva, David, Eyal, sans oublier mon cousin Robert et Jeannine.

A Claude et Audrey Micallef, de nous avoir reçu comme leur famille.

A mes amis,

A mes amis de longue date : Mélanie et Isaac, Keren, Cynthia, Déborah, Yohann, Benjamin, Laurent, Jonathan et Dana, Michael et Sarah, Johanna et Yohan R, Johanna et Yohann D, Johanna et Kevin, Abigaeuuullle, Anaëlle et llann...

A mes potes de promo: Bob mon duo de sous-colle, Simon, Mike, Aude et Alex, Chimon, Lionel, Elie (pour notre stage fantôme en MPR), Anaïs mon binôme de garde, Sam Co, Idir, Vincent Jerem et Adrien pour les ambiances en stage.

A mes amis niçois : Jacob et Manue de nous avoir fait découvrir le reste de la liste, Anthony, Gab et Rachel, Judicael et Judith, Israël et Léa, David et Jordan, Anthony

A mes cousins niçois Alex et llana, vous formez un couple magnifique.

\section{A ceux non encore cités,}

A la cellule de programmation mais surtout à Yamina, pour ton efficacité.

A l'équipe de Biostatistiques du CAL, Antoine, Jocelyn et Yann, pour votre motivation et votre efficacité. Ce travail n'aurait pu exister sans votre aide.

A Eugénia MARINE-BARJOAN, qui a pris le temps de m'expliquer les statistiques, de faire et refaire mes analyses.

Aux patients qui rendent notre métier passionnant.

Aux étudiants exigeants de la faculté de Nice qui me poussent à m'améliorer chaque jour. 


\title{
A mon noyau familial,
}

\begin{abstract}
A Dorine,
Voilà maintenant plus de dix ans que l'on se tient la main... ces quelques mots ne suffiront donc jamais à exprimer les remerciements que je te dois et l'amour que je te porte. Nous sommes tellement complices que je pourrais te faire rentrer dans quasiment toutes les cases de ces remerciements : bien sûr la famille, mais aussi les collègues, et encore plus les amis. Toi, ma moitié, avec qui dix ans après, je ne me lasse pas de rester à discuter pendant des heures comme si on ne s'était pas vu pendant des mois. Notre relation est volcanique, un bouillonnement d'émotions qui mélange l'amour, l'amitié, le respect, la franchise et des valeurs communes. Tu as contribué à tout ce que j'ai touché ces dix dernières années, tu m'as aidé dès la première année de médecine puis tu as continué jour après jour à m'encourager, m'entraîner, me pousser toujours plus haut. Pendant l'externat tu as appris chaque mot, chaque cours, chaque mnémotechnique avec moi parfois jusqu'à des horaires pas possibles. Tu as travaillé pendant mes études pour que l'on puisse se marier et tu as assumé les revenus de la famille. Puis tu as tout quitté, famille, amis et travail, pour me suivre loin de chez nous, afin que je puisse exercer la spécialité que j'avais choisi. Pendant l'internat tu as continué à me motiver malgré les horaires incroyables que je faisais, tu me poussais à aller au bout de ma passion. Tu as partagé mes joies et mes peines comme si c'était les tiennes. Tu t'es occupée de tout ce que j'aurais pu faire pour que je puisse me concentrer sur mon travail. Tu as d'ailleurs aussi contribué à celui que je présente ce soir. Aujourd'hui je pense que tu mérites de recevoir mes remerciements, mais que tu peux également prendre pour toi tous les compliments qui me sont adressés...
\end{abstract}

\section{A Emmy,}

Ma fille, mon ainée, mon bébé, il est trop tôt pour que tu comprennes ce que je t'écris aujourd'hui, mais tu le liras surement un jour. Merci d'être ma motivation chaque jour. Merci pour l'amour que tu nous portes à ta mère et moi. Nous sommes déjà très fiers de toi alors que tu n'as que trois ans, et j'ai toute confiance pour que tu continues à nous étonner. Tu es et tu resteras ce que j'ai de plus cher dans ma vie. Reste toujours pleine de vie comme tu l'es. Je te promets que ta mère et moi ferons tout pour t'élever afin que tu deviennes une femme épanouie et responsable.

\section{A ma deuxième fille,}

Tu n'es pas encore mais nous t'attendons avec impatience. Nous pensons à toi chaque jour (surtout ta mère quand tu tapes dans sa vessie) et t'aimons déjà très fort. 


\section{RESUME}

Introduction : La thermoablation percutanée (TAP) pour les patients porteurs de carcinomes hépatocellulaires $(\mathrm{CHC})$ oligonodulaires ou uninodulaires entre 30 et $50 \mathrm{~mm}$ peut être insuffisante. Son association synchrone à un traitement endoartériel (TE) lipiodolé avec ou sans embolisation complémentaire a été proposée pour potentialiser son efficacité. Le but de cette étude était d'évaluer l'efficacité et la tolérance d'un traitement combiné par TAP et TE réalisé en une seule séance pour des patients porteurs de $\mathrm{CHC}$ dans les critères de Milan.

Patients et Méthodes : Analyse rétrospective monocentrique d'un groupe de 30 patients consécutifs ayant eu un premier traitement combiné entre janvier 2009 et décembre 2014 pour un $\mathrm{CHC}$ histologiquement prouvé, uninodulaire, entre 30 et $50 \mathrm{~mm}$ ou oligonodulaire ( $\mathrm{n}$ $\leq 3$ ) de moins de $30 \mathrm{~mm}$ ( " groupe TAP+TE »). Un groupe témoin était constitué de 34 patients consécutifs avec un $\mathrm{CHC}$ uninodulaire de moins de $30 \mathrm{~mm}$ sans aucun critère de mauvais pronostic en imagerie (caractère infiltrant, micronodules satellites, extension tumorale endoportale) traités par TAP seule (« groupe TAP »). L'objectif principal était l'étude de la survie globale (SG).

Résultats : La majorité des patients étaient cirrhotiques (90\% vs $91 \%$ respectivement dans les groupes TAP+TE vs TAP), avaient un score Performans Status à $0(80 \%$ vs $79 \%)$ et une tumeur bien différenciée (53\% vs $56 \%$ ). Parmi les patients cirrhotiques la majorité étaient CHILD A ( $83 \%$ vs $85 \%$ ). Les patients du groupe TAP+TE présentaient moins fréquemment une hypertension portale ( $47 \%$ vs $76 \%, p=0,03$ ). II n'y avait pas de différence significative du taux médian d'alpha-fœto-protéine entre les 2 groupes (15 vs 9,3 $\mu g / l, p=0,39)$.

Aucun échec de procédure n'a été observé. Une réponse complète était observée en imagerie dans $93 \%$ vs $97 \%$ respectivement dans les groupes TAP+TE vs TAP $(p=0,91)$. La SG à 1, 3 et 5 ans était significativement identique dans les 2 groupes : $100 \%, 82 \%$ et $48,5 \%$ dans le groupe TAP+TE et $94,1 \%, 72,7 \%$ et $65,2 \%$ dans le groupe TAP $(p=0,88)$. La survie sans récidive à 1,3 et 5 ans était significativement plus basse dans le groupe TAP+TE : $59,3 \%, 18,5 \%$ et $5,6 \%$, contre $89,3 \%, 46,4 \%$ et $24,5 \%$ dans le groupe TAP ( $p$ $=0,02)$. Des complications sont survenues chez respectivement $27 \%$ et $15 \%$ des patients $(p=0,38)$ dans les groupes TAP+TE et TAP, majoritairement des douleurs grade $\mathrm{I} / \mathrm{II}(13 \%$ et $9 \%, p=0,75)$, et uniquement 2 complications grade III dans chaque groupe $(p=0,47)$. II n'y avait pas de différence sur la proportion de patients transplantés dans les 2 groupes (20 $\%$ vs $12 \%, p=0,58)$.

Conclusion : L'association d'un traitement TAP+TE, chez des patients ayant un CHC entre 30 et $50 \mathrm{~mm}$ ou oligonodulaires $(\mathrm{n} \leq 3)$ de moins de $30 \mathrm{~mm}$, permettrait d'avoir une survie globale similaire à celle de patients ayant un $\mathrm{CHC}$ uninodulaire de moins de $30 \mathrm{~mm}$ sans critère de mauvais pronostic traités par TAP, sans plus de complications mais avec une récidive tumorale plus précoce. 


\section{Endoarterial treatment in combination with percutaneous thermoablation for medium-sized and oligonodular hepatocellular carcinomas}

Dann Joseph Ouizeman ${ }^{1}$, Antoine Falcoz ${ }^{2}$, Emmanuel Chamorey², Yann Chateau², Jocelyn Gal $^{2}$, Edouard Larrey ${ }^{1}$, Thierry Piche ${ }^{1,3}$, Albert Tran $^{1,3,4}$, Patrick Chevallier ${ }^{3,5}$, Rodolphe Anty ${ }^{1,3,4}$

1. University Hospital of Nice, Digestive Center, Nice, France

2. Centre Antoine Lacassagne, Department of Epidemiology and Biostatistics, Nice, France

3. University Côte d'Azur, Faculty of Medicine, Nice, France

4. National Institute of Health and Medical Research (INSERM), U1065, C3M, Team 8 "Chronic liver diseases associated with obesity and alcohol », Nice, France

5. University Hospital of Nice, Department of Diagnostic and Interventional Imaging, Nice, France

Word count of main body of manuscript: 3288

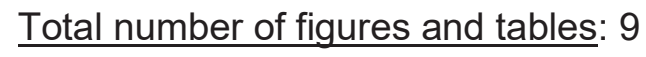

List of abbreviations (in order of appearance):

HCC, Hepatocellular carcinoma; PT, Percutaneous thermoablation; RFA, Radiofrequency ablation; TAC, Transarterial chemotherapy; OS, Overall survival; RFS, Relapse free survival; CTCAE, Common Terminology Criteria for Adverse Events; HR, Hazard ratio; IC95, 95\% confidence interval; PS, Performans status; MWA, Microwave ablation; DC-BEADS, Drugeluting beads; BCLC, Barcelona Clinic Liver Cancer

None of the authors have any commercial association or other conflicts of interest regarding the manuscript. No financial support was received for the study. 


\section{ABSTRACT}

Background \& Aims: The association of transcatheter arterial chemotherapy infusion (TAC) with percutaneous ablation (PT) has been introduced as a method to increase the size of the ablative zone and treat satellites micronodules. The aim of this study was to evaluate the efficacy of PT and TAC performed in a single session for hepatocellular carcinomas (HCCs) between 30 and $50 \mathrm{~mm}$ or oligonodular $\mathrm{HCC}$ up to $30 \mathrm{~mm}$.

Methods: Thirty patients with a histologically proven HCC, uninodular between 30 and $50 \mathrm{~mm}$ or oligonodular $(n \leq 3)$ up to $30 \mathrm{~mm}$, treatment-naïve and non-metastatic, received the combination treatment ("PT+TAC group"). A "control" group consisted of 34 patients with uninodular HCC up to $30 \mathrm{~mm}$ without any poor prognostic criteria treated with PT alone ("PT group").

Results: The majority of patients were cirrhotic ( $90 \%$ vs $91 \%$, respectively for the PT+TAC and PT groups), Child-Pugh A ( $83 \%$ vs $85 \%)$, with a Performans Status of 0 ( $80 \%$ vs $79 \%)$. There was no significant difference in the median alpha-fetoprotein level (15 vs $9.3 \mu \mathrm{g} / \mathrm{l}$, $\mathrm{p}=0.39$ ). The 1-, 3-, and 5-year OS was no significantly different in both groups: $100 \%, 82 \%$ and $48.5 \%$ in the PT+TAC group vs $94.1 \%, 72.7 \%$ and $65.2 \%$ in the PT group ( $p=0.88)$. The 1-, 3-, and 5-year relapse-free survival was significantly lower in the PT+TAC group: 59.3\%, $18.5 \%$ and $5.6 \%$ vs $89.3 \%, 46.4 \%$ and $24.5 \%$, in the PT group ( $p=0.02)$.

Conclusion: The combination treatment for HCC between 30 and $50 \mathrm{~mm}$ or oligonodular up to $30 \mathrm{~mm}$, showed a similar OS, compared to uninodular HCC up to $30 \mathrm{~mm}$ without poor prognostic criteria, treated with PT alone, at the price of earlier tumor recurrence.

Keywords: Hepatocellular carcinoma; Transarterial chemoembolization; Radiofrequency ablation; Microwave ablation

Lay Summary: New curative treatments for liver cancer are under development. The combination of thermal ablation and arterial chemotherapy infusion is a promising therapeutic avenue. The combination of these treatments performed in a single session shows survival equal to that of smaller liver cancers, without more complications. However, these initially larger cancers recur more often and require more additional treatment. 


\section{INTRODUCTION}

Hepatocellular carcinoma (HCC) accounts for $7 \%$ of all cancers worldwide. With about 800.00 cases per year it is the sixth most common tumor, while with 780.000 death per year it is the fourth most frequent cause of death from cancer in $2019^{1}$. In France, the most recent data show a net survival at 1,3 and 5 years respectively of $46 \%, 23 \%$ and $15 \%^{2}$. Despite the implementation of surveillance programs, many patients are detected at an intermediate or advanced stage of $\mathrm{HCC}^{3}$, and only $23 \%$ of diagnosed patients have access to curative treatment ${ }^{4}$.

Percutaneous thermoablation (PT) is recommended by French, American and Asian societies as curative treatment for $\mathrm{HCC} \leq 30 \mathrm{~mm}^{5-8}$. During radiofrequency ablation (RFA), the heat propagates in a centrifugal direction from the energy source (electrode tip) in the center of the tumor to the periphery of the tumor (« centrifugal » ablation) and the temperature decreases, together with the distance from the electrodes and when the blood flow is present in the vicinity. This phenomenon explains the decrease in local control of a tumor larger than 20 to $30 \mathrm{~mm}$, as well as the decrease in efficacy of the technique when the tumor is localized near a major vessel (the so-called « heat sink effect $»)^{9}$. Surgical resection is recommended for HCC between 30 and $50 \mathrm{~mm}$ or for $\mathrm{HCC}$ with 2 to 3 nodules located in the same segment ${ }^{5,8}$. Only 5 to $40 \%$ of HCCs are candidates for surgical treatment because of inappropriate localization, presence of portal hypertension, inappropriate hepatic function or excessive comorbidity 5,10 . HCCs between 30 and $50 \mathrm{~mm}$ and oligonodular $(n \leq 3)$ are at higher risk of recurrence. PT for these nodules leads to a complete necrotic rate of 29 to $70 \%{ }^{11}$. This population of HCC, in case of the absence of accessibility to a surgical treatment, benefits from non-curative treatment by transcatheter arterial chemotherapy infusion with or without embolization (TAC). Preliminary studies suggest that the association of TAC with PT leads to better overall survival and recurrence-free survival ${ }^{12}$. The combination in a single session has been introduced as a method to increase the size of the ablative zone because TAC can decrease perfusionmediated heat loss during RFA ${ }^{13}$ if TAC is performed first. If PT is performed first a physiologic 
hyperhemia rim created around the hyperthermia zone increases the effect of the TAC by leading an accumulation of drug and embolic material within this rim. Furthermore, the association of a TAC can treat satellites micronodules which can be far away the primitive tumor. This method has been studied mainly in Asian populations. It has been shown to be effective for HCCs greater than $50 \mathrm{~mm}^{14}$ and for HCCs less than $30 \mathrm{~mm}$ infeasible for ultrasound-guided RFA ${ }^{15}$.

The objective of our study was to evaluate the efficacy and safety of TAC and PT performed in a single session for a European population with treatment-naïve HCC between 30 and 50 $\mathrm{mm}$ or oligo-nodular up to $30 \mathrm{~mm}$ compared to uninodular HCC up to $30 \mathrm{~mm}$ without any poor prognostic criteria treated with PT alone.

\section{PATIENTS AND METHODS}

\section{$\underline{\text { Selection of patients }}$}

This was a retrospective cohort study based on information collected prospectively from the medical records at the University Hospital of Nice, France. Between January 2009 and December 2014, we identified patients with histologically proven HCC who received, in firstline treatment, a combination of TAC and PT in a single session. Among them, we selected those with one nodule between 30 and $50 \mathrm{~mm}$ or 2 to 3 nodules up to $30 \mathrm{~mm}$ for the "PT+TAC group". Patients with a discordant arteriography and extra-hepatic metastasis were excluded. A "control" group of uninodular HCCs up to $30 \mathrm{~mm}$ without any poor prognostic criteria (infiltrating character, satellite micronodules, endoportal tumor extension) was identified and treated by PT alone. For the "PT group" patients with histologically proven HCC who received PT in first-line treatment and without extra-hepatic metastasis were selected. The study complied with the provisions of the Declaration of Helsinki and was approved by the Ethics Committee of our institution (Authorization reference no. 319). 


\section{Methods}

All procedures were performed under general anesthesia under conditions of surgical asepsis. For the PT+TAC group, pre-ultrasound and arteriography were performed, followed by PT and TAC in a single session.

PT procedure: A radiofrequency (Boston scientific) or microwave (Amica) needle was deployed percutaneously under sonographic guidance. Several applications were carried out if necessary (mean of 1.8 applications).

TAC procedure: TAC was performed immediately after PT in the same session. A 4-French introducer was inserted in the femoral artery using the Seldinger technique. A coeliomesenteric arteriography was performed to investigate the site and size of the HCCs and to confirm patency of the portal vein. A selective or hyper-selective catheterization of a branch of the hepatic artery was then followed by injection of a solution of chemotherapy $(1 \mathrm{mg} / \mathrm{kg}$ doxorubicine or adriamycin) mixed with $10 \mathrm{ml}$ of iodized oil (Lipiodol ultrafluide, Guerbet, France), with or without a complementary embolization (embozenes $250 \mu$, Boston scientific). The selectivity of the TAC was chosen during the procedure on the basis of the tumoral burden. The technical goal was to obtain blood stasis in the treated liver for 3 to 5 heart beats during the final arteriography.

\section{Assessment and follow-up}

The primary endpoint was the cumulative overall survival (OS) rate from initial treatment until the last follow-up or death. The secondary endpoints were the relapse free survival (RFS) rate from initial treatment until first relapse or death, and complications as described in the Common Terminology Criteria for Adverse Events (CTCAE) version 5.0. We also assessed the technical feasibility and therapeutic response on the first follow-up imagery.

Computed tomography or magnetic resonance imaging of the liver was performed in a mean of 5.2 weeks after the therapeutic session. The morphological follow-up was left to the 
discretion of the practitioners, but usually every 3 to 4 months the first year and every 6 months thereafter. In case of tumoral recurrence, the most appropriate therapy was given according to clinical guidelines.

\section{Statistical analysis}

Distribution of continuous and categorical variables were summarized as mean (standard deviation) or median values ( $\min$ and $\max$ ), and frequencies (percentage), respectively. Medians and proportions were compared using the Wilcoxon test and $\mathrm{x} 2$ test (or Fisher's exact test, if appropriate), respectively. OS and RFS curves were estimated with the Kaplan-Meier method and compared between the PT and PT+TAC groups using the log-rank test. Cox proportional hazard models were performed to estimate the hazard ratio (HR) and the 95\% confidence interval (IC95) for factors associated with OS or RFS. The association of parameters with OS or RFS was first assessed using univariate Cox analyses and then those with $p<0.1$ were entered into a final multivariable Cox regression model including the treatment group. The collinearity between variables entered into the model was evaluated using the ' $r$ ' Pearson correlation coefficient. If $r>0.40$, one of the two variables was considered as redundant and was removed from the model. Finally, a stepwise selection was performed, forcing the treatment group variable in the final model. Complications were assessed in both groups and compared using the $x^{2}$ test or Fisher's exact test, if appropriate. All analyses were performed using $R$ version 3.5.1. Values of $p<0.05$ were considered statistically significant and all tests were two-sided.

\section{RESULTS}

Among the 151 patients who received a combination of TAC and PT in a single session in the period considered, 61 had previous treatment for HCC (surgery, transplantation, TAC, PT or systemic chemotherapy), 40 had nodules outside the size criteria, 11 did not have HCCs, 5 had no histological evidence, 3 had a discordant arteriography and 1 was metastatic. Finally, 30 consecutive patients were included in the PT+TAC group (Figure 1a). Among the 557 
patients who received PT alone in the period considered, 94 had a HCC without any prior treatment. Among them, 41 had nodules outside the size criteria, 12 had no histological evidence, 6 had poor prognostic criteria (4 had endoportal tumor extension and 2 had satellite micronodules) and 1 was metastatic. Finally, 34 consecutive patients were included in the PT group (Figure 1b).

\section{Baseline characteristics}

There was no significant difference between the mean age in both groups: 65.1 years vs 66.2 years, respectively for the PT+TAC and PT groups $(p=0.724)$, nor in the sex ratio $(p=0.381)$, see Table 1. The histological differentiation was similar in both groups: HCC was welldifferentiated for $53.3 \%$ vs $55.9 \%$, respectively $(p=1)$. Ninety percent of the population was cirrhotic without a significant difference between the two groups $(p=1)$, and $63.0 \%$ and $64.5 \%$, respectively were Child-Pugh A5 $(p=1)$. Eighty percent of the population had a Performans Status (PS) score of 0 , without a significant difference between the two groups $(p=1)$. There was no significant difference in etiology between the two groups: alcoholic for $40.0 \%$ vs $50.0 \%$, respectively $(p=0.582)$, hepatitis $C$ virus for $70.0 \%$ vs $47.1 \%$, respectively $(p=0.109)$, hepatitis B virus for $3.3 \%$ vs $11.8 \%$, respectively ( $p=0.431$ ), and non-alcoholic steatohepatitis for $6.7 \%$ vs $8.8 \%$, respectively $(p=1)$. Portal hypertension, endoscopically or imaging highlighted, was significantly less present in the PT+TAC group (46.7\%) vs the PT group $(76.5 \%, p=0.028)$. There was no significant difference between the two groups for the median alpha-foetoprotein level: 15.0 vs $9.3 \mu \mathrm{g} / \mathrm{l}$, respectively in the PT+TAC vs PT group $(p=0.393)$. There was no significant difference between the two groups for the patient's major comorbidity $(p=0.471)$. Major comorbidities were principally other neoplasia or cardiopulmonary conditions, which precluded surgical management.

In the PT+TAC group, the thermoablation was performed by RFA for $66.7 \%$ and by microwave ablation (MWA) for $33.3 \%$. In the PT group, the distribution was equal in the two groups $(p=0.274)$. 
The chemotherapy product used for TAC was Adriamycin for $60 \%$ and Doxorubicin for $40 \%$.

Drug-eluting beads (DC-BEADS) were used for $13.3 \%$ of patients.

Figure 1a: Flow chart of the PT+TAC group

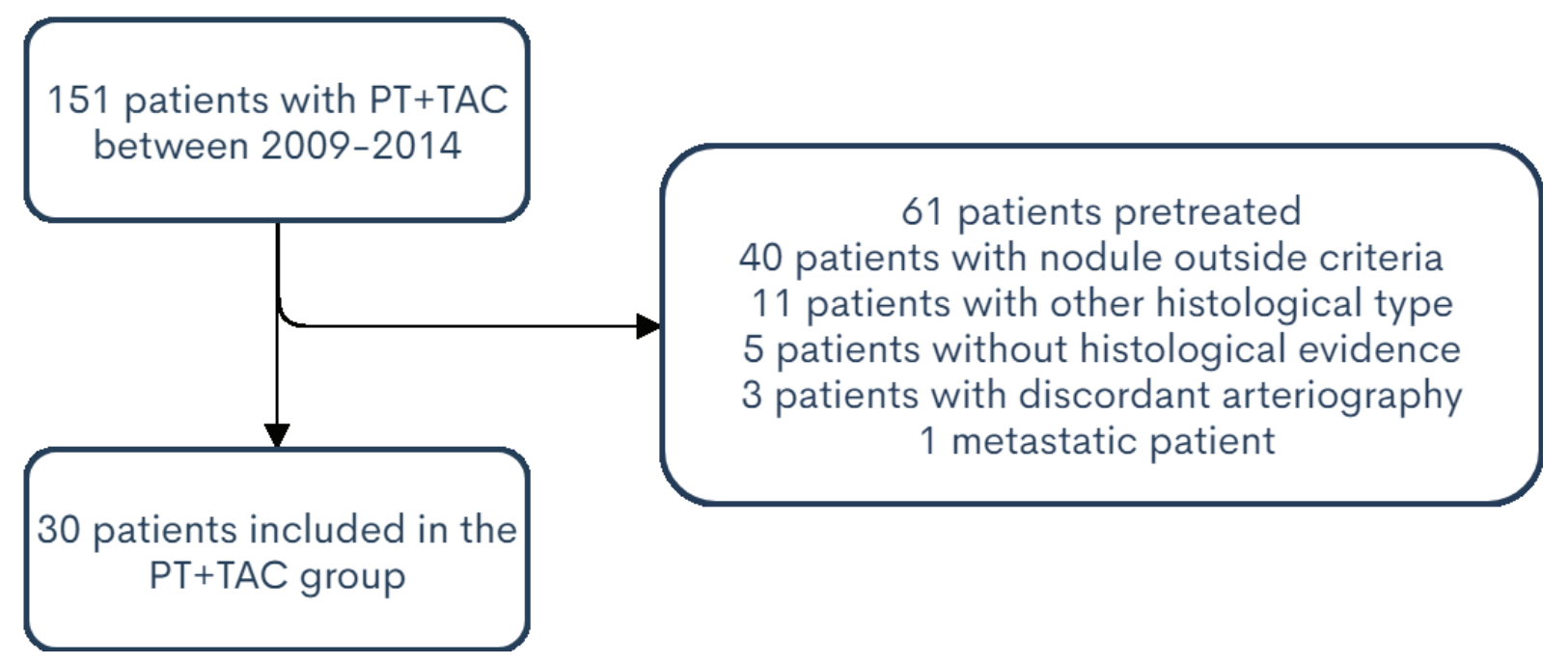

Figure 1b: Flow chart of the PT group

557 patients with PT alone between 2009-2014

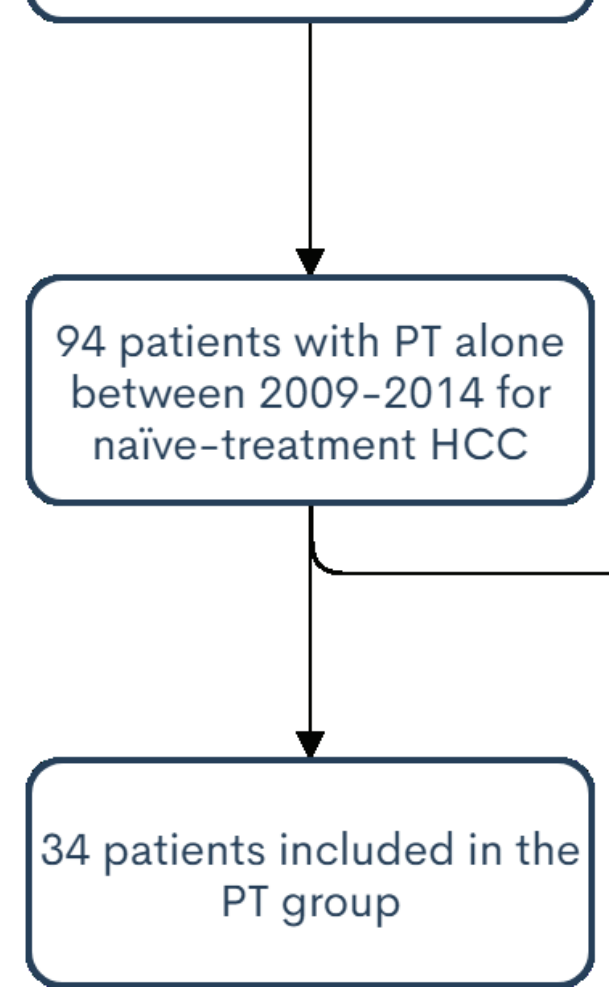

41 patients with nodule outside criteria 12 patients without histological evidence 1 metastatic patient 6 patients with pejorative criteria 
Table 1: Patient and tumor characteristics

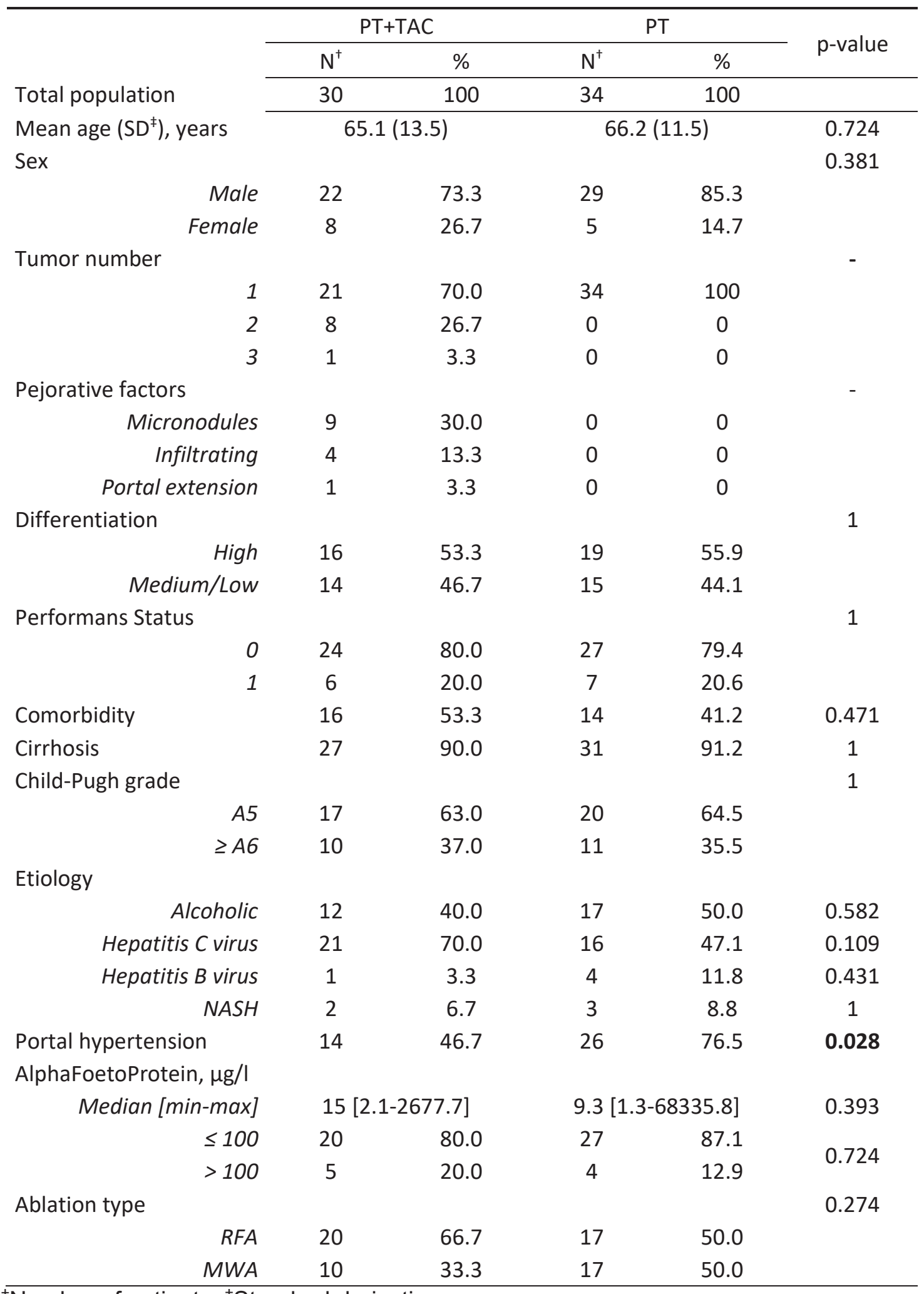

${ }^{\dagger}$ Number of patients, ${ }^{\ddagger}$ Standard derivation 


\section{$\underline{\text { Overall survival }}$}

No procedural failure was identified. Complete response evaluated on the first follow-up imaging was noted in $93 \%$ vs $97 \%$, respectively for the PT+TAC and PT groups $(p=0.91)$.

The 1-, 3-, and 5-year OS was $100 \%, 82 \%$ and $48.5 \%$, respectively, in the PT+TAC group, which was significantly identical to the PT group: $94.1 \%, 72.7 \%$ and $65.2 \%$ ( $p=0.88$, Figure 2 ). In a univariate analysis, factors with a negative impact on the OS were the Child-Pugh score $\geq \mathrm{A} 6(\mathrm{HR}=2.8 ; \mathrm{IC95}$ [1.2 to 6.4]; $p=0.017)$, and PS = $1(\mathrm{HR}=3.0 ; \mathrm{IC} 95$ [1.3 to 7.1]; $p=0.011)$ see Table 2. In a multivariate analysis, these factors remained significant: for the Child-Pugh score $\geq A 6(H R=2.96 ; \operatorname{IC95}[1.2$ to 7.6$] ; p=0.023)$, and PS = $1(H R=4.7 ; \operatorname{IC95}$ [1.8 to 12.6]; $p=0.002$ ), see Table 2. The absence of a significant difference in the OS between the two groups was underlined by a non-significant HR of PT+TAC vs PT (HR = 0.9; IC95 [0.4 to 2.3]; $\mathrm{p}=0.833)$.

There was no difference in the proportion of patients transplanted in both groups: $20 \%$ in the PT+TAC group vs $12 \%$ in the PT group $(p=0.58)$.

\section{$\underline{\text { Relapse free survival }}$}

The 1-, 3-, and 5-year RFS was 59.3\%, 18.5\% and 5.6\% in the PT+TAC group, which was significantly lower than in the PT group: $89.3 \%, 46.4 \%$ and $24.5 \%(p=0.02$, Figure 3$)$. In a univariate analysis, the only factor with a negative impact on the RFS was the PT+TAC treatment $(H R=1.9 ;$ IC95: [1.1 to 3.4$] ; p=0.023)$, see Table 3 . In a multivariate analysis, this result was not significant (Table 3).

There was significantly more uninodular relapse in the PT+TAC group than in the PT group: $66 \%$ vs $50 \%$ respectively $(p=0.029$ ), see Table 4 . The maximal tumor size on relapse was significantly identical in both groups: mean of $22.4 \mathrm{~mm}$ vs $18.6 \mathrm{~mm}$, respectively in the PT+TAC and PT group $(p=0.467)$. 
Patients of the PT+TAC group were significantly more re-treated than in the PT group (Table 5). One and 3 years after initial treatment $56.7 \%$ and $63.3 \%$ of the PT+TAC group was treated again, vs $14.7 \%$ and 32.3 of the PT group ( $p=0.003$ and $p=0.001$, respectively). There was no significant difference at 5 years.

Figure 2: Kaplan-Meier curves of overall survival comparing PT+TAC group and PT group

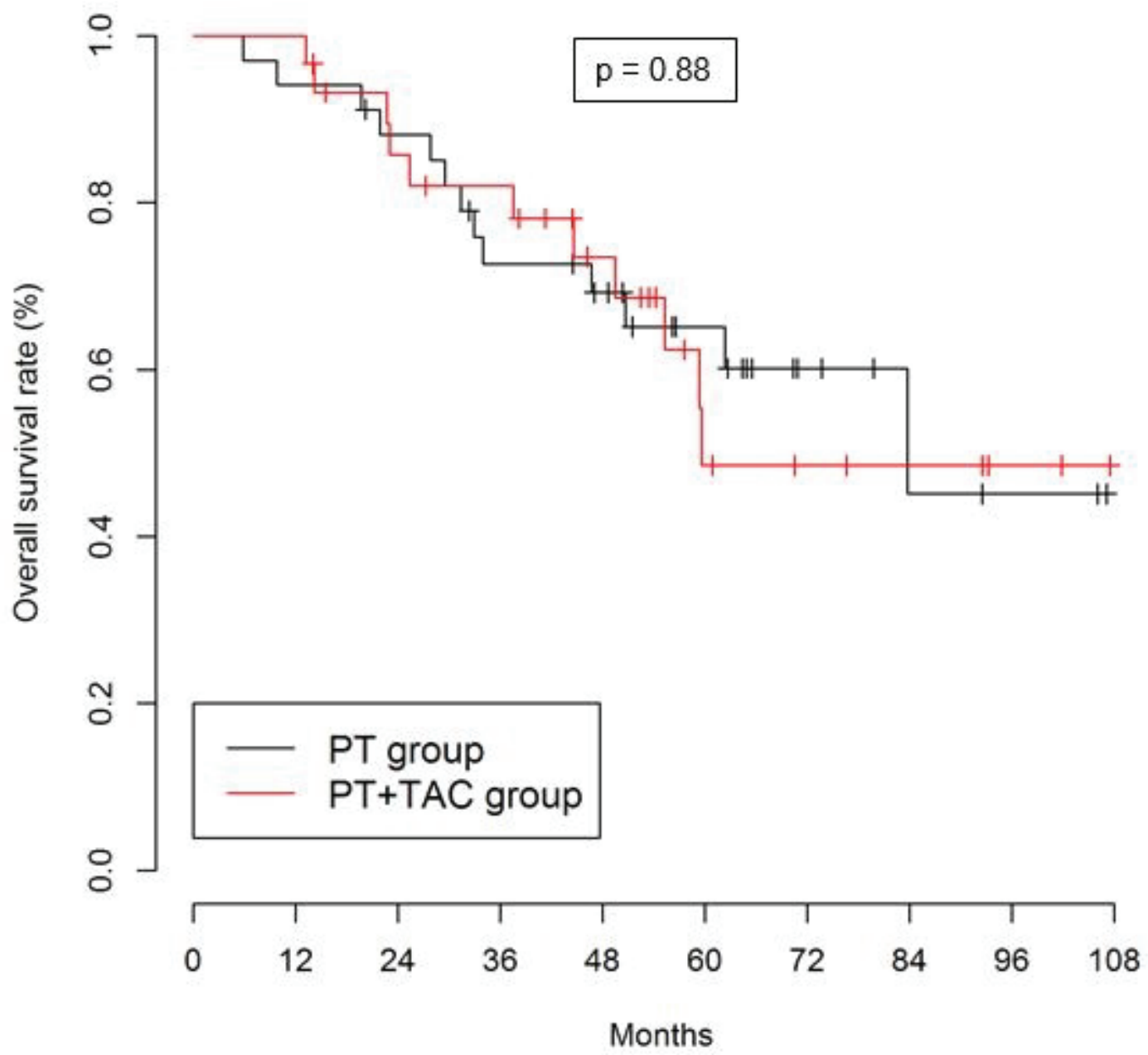

There is not significant difference on overall survival between the two groups. 
Table 2: Univariate and multivariate overall survival analysis

\begin{tabular}{|c|c|c|c|c|c|c|}
\hline & \multicolumn{3}{|c|}{ Univariate analysis } & \multicolumn{3}{|c|}{ Multivariate analysis } \\
\hline & $\mathrm{HR}^{+}$ & $\mathrm{IC}_{95 \%}{ }^{\ddagger}$ & $\mathrm{p}$ & $\mathrm{HR}^{+}$ & $\mathrm{IC}_{95 \%}{ }^{\ddagger}$ & $\mathrm{p}$ \\
\hline \multicolumn{7}{|l|}{ Group } \\
\hline$P T$ & & Reference & & & Referenc & \\
\hline$P T+T A C$ & 1.1 & $0.5-2.4$ & 0.880 & 0.9 & $0.4-2.3$ & 0.833 \\
\hline \multicolumn{7}{|l|}{ Age, years } \\
\hline$<70$ & & Reference & & & & \\
\hline$\geq 70$ & 0.7 & $0.3-1.6$ & 0.356 & & & \\
\hline \multicolumn{7}{|l|}{ Differentiation } \\
\hline High & & Reference & & & & \\
\hline Medium/Low & 1.1 & $0.5-2.4$ & 0.896 & & & \\
\hline \multicolumn{7}{|l|}{ Performans Status } \\
\hline 0 & & Reference & & & Referenc & \\
\hline 1 & 3.0 & $1.3-7.1$ & 0.011 & 4.7 & $1.8-12.6$ & 0.002 \\
\hline \multicolumn{7}{|l|}{ Comorbidity } \\
\hline No & & Reference & & & & \\
\hline Yes & 1.9 & $0.9-4.4$ & 0.114 & & & \\
\hline \multicolumn{7}{|l|}{ CHILD score } \\
\hline A5 & & Reference & & & Referenc & \\
\hline$\geq A 6$ & 2.8 & $1.2-6.4$ & 0.017 & 3.0 & $1.2-7.6$ & 0.023 \\
\hline \multicolumn{7}{|l|}{ Portal hypertension } \\
\hline No & & Reference & & & & \\
\hline Yes & 1.9 & $0.8-4.8$ & 0.170 & & & \\
\hline \multicolumn{7}{|l|}{ AlphaFoetoProtein, $\mu \mathrm{g} / \mathrm{l}$} \\
\hline$\leq 100$ & & Reference & & & & \\
\hline$>100$ & 1.9 & $0.6-5.8$ & 0.249 & & & \\
\hline \multicolumn{7}{|l|}{ Ablation type } \\
\hline$R F A$ & & Reference & & & & \\
\hline MWA & 0.8 & $0.4-1.9$ & 0.648 & & & \\
\hline
\end{tabular}


Figure 3: Kaplan-Meier curves of relapse free survival comparing PT+TAC group and PT group

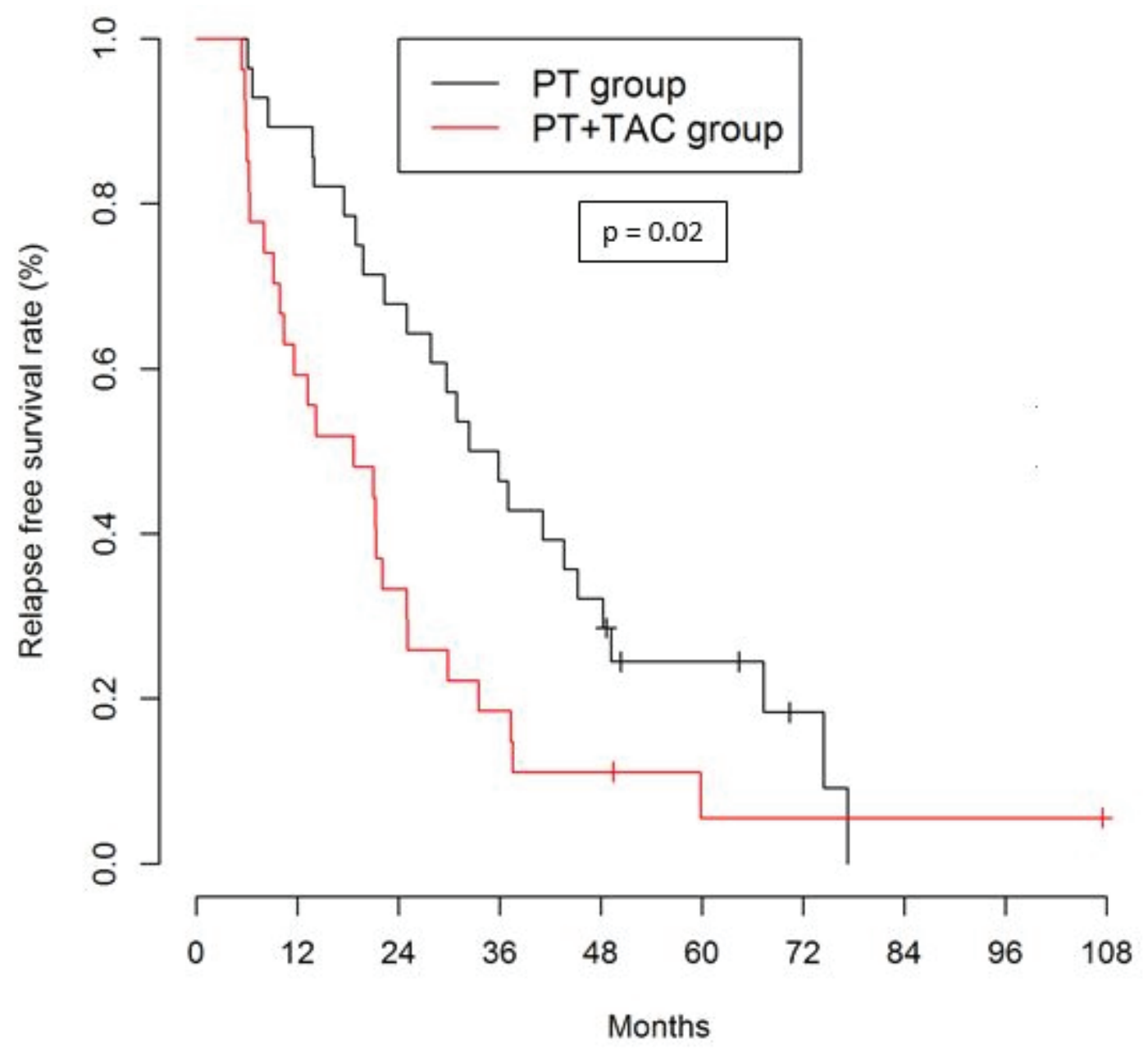

The relapse was significantly faster in the PT+TAC group than in the PT group. 
Table 3: Univariate and multivariate relapse free survival analysis

\begin{tabular}{|c|c|c|c|c|c|c|}
\hline & \multicolumn{3}{|c|}{ Univariate analysis } & \multicolumn{3}{|c|}{ Multivariate analysis } \\
\hline & $\mathrm{HR}^{+}$ & $\mathrm{IC}_{95 \%}{ }^{\ddagger}$ & $\mathrm{p}$ & $\mathrm{HR}^{+}$ & $\mathrm{IC}_{95 \%}{ }^{\ddagger}$ & $\mathrm{p}$ \\
\hline \multicolumn{7}{|l|}{ Group } \\
\hline PT & & Reference & & & Referen & \\
\hline$P T+T A C$ & 1.9 & $1.1-3.4$ & 0.023 & 1.5 & $0.8-2.9$ & 0.185 \\
\hline \multicolumn{7}{|l|}{ Age, years } \\
\hline$<70$ & & Reference & & & & \\
\hline$\geq 70$ & 0.8 & $0.5-1.5$ & 0.509 & & & \\
\hline \multicolumn{7}{|l|}{ Differentiation } \\
\hline High & & Reference & & & & \\
\hline Medium/Low & 1.1 & $0.6-1.9$ & 0.841 & & & \\
\hline \multicolumn{7}{|l|}{ Performans Status } \\
\hline 0 & & Reference & & & & \\
\hline 1 & 1.0 & $0.5-2.1$ & 0.904 & & & \\
\hline \multicolumn{7}{|l|}{ Comorbidity } \\
\hline No & & Reference & & & & \\
\hline Yes & 0.8 & $0.4-1.4$ & 0.418 & & & \\
\hline \multicolumn{7}{|l|}{ CHILD score } \\
\hline A5 & & Reference & & & & \\
\hline$\geq A 6$ & 1.4 & $0.8-2.7$ & 0.271 & & & \\
\hline \multicolumn{7}{|l|}{ Portal hypertension } \\
\hline No & & Reference & & & & \\
\hline Yes & 0.9 & $0.5-1.6$ & 0.673 & & & \\
\hline \multicolumn{7}{|c|}{ AlphaFoetoProtein, $\mu \mathrm{g} / \mathrm{l}$} \\
\hline$\leq 100$ & & Reference & & & & \\
\hline$>100$ & 1.7 & $0.8-4.0$ & 0.190 & & & \\
\hline \multicolumn{7}{|l|}{ Ablation type } \\
\hline RFA & & Reference & & & & \\
\hline$M W A$ & 1.0 & $0.5-1.7$ & 0.854 & & & \\
\hline
\end{tabular}


Table 4: Relapse

\begin{tabular}{|c|c|c|c|c|c|}
\hline & \multicolumn{2}{|c|}{ PT+TAC } & \multicolumn{2}{|c|}{ PT } & \multirow{2}{*}{$p$-value } \\
\hline & $\mathrm{N}^{+}$ & $\%$ & $\mathrm{~N}^{+}$ & $\%$ & \\
\hline Total relapse & 23 & 76.7 & 24 & 85.7 & 0.700 \\
\hline Hepatic relapse & & & & & 0.023 \\
\hline 1 & 15 & 65.2 & 12 & 50.0 & \\
\hline 2 & 6 & 26.1 & 2 & 8.3 & \\
\hline 3 & 0 & 0 & 6 & 25 & \\
\hline $\begin{array}{r}\text { Multinodular }(\geq 4) \text { or } \\
\text { infiltrating }\end{array}$ & 2 & 8.7 & 1 & 4.2 & \\
\hline Metastatic & 0 & 0 & 3 & 12.5 & 0.234 \\
\hline
\end{tabular}

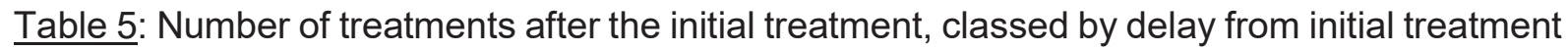
for alive patients

\begin{tabular}{|c|c|c|c|c|c|c|}
\hline \multirow{2}{*}{\multicolumn{2}{|c|}{$\begin{array}{l}\text { Delay from initial } \\
\text { treatment }\end{array}$}} & \multicolumn{2}{|c|}{$P T+T A C$} & \multicolumn{2}{|c|}{ PT } & \multirow{2}{*}{$\mathrm{p}$-value } \\
\hline & & $\mathrm{N}^{+}$ & $\%$ & $\mathrm{~N}^{+}$ & $\%$ & \\
\hline \multirow[t]{4}{*}{1 year } & & & & & & 0.003 \\
\hline & 0 & 13 & 43.3 & 27 & 79.4 & \\
\hline & 1 & 11 & 36.7 & 3 & 8.8 & \\
\hline & $\geq 2$ & 6 & 20 & 2 & 5.9 & \\
\hline \multirow[t]{4}{*}{3 years } & & & & & & 0.001 \\
\hline & 0 & 2 & 6.7 & 12 & 35.3 & \\
\hline & 1 & 6 & 20 & 8 & 23.5 & \\
\hline & $\geq 2$ & 13 & 43.3 & 3 & 8.8 & \\
\hline \multirow[t]{4}{*}{5 years } & & & & & & 0.164 \\
\hline & 0 & 0 & 0 & 5 & 14.7 & \\
\hline & 1 & 2 & 6.7 & 2 & 5.9 & \\
\hline & $\geq 2$ & 5 & 16.7 & 6 & 17.7 & \\
\hline
\end{tabular}

${ }^{\dagger}$ Number of patients 


\section{$\underline{\text { Complications }}$}

Complications occurred in $27 \%$ and $15 \%$ of patients $(p=0.38)$, respectively for the PT+TAC and PT groups; including mostly pain grade I/II according to CTCAE (50\% and 60\%, respectively, $p=0.75$, Table 6 ). Other complications in the PT+TAC group included: 2 liver bleedings, none requiring transfusion ( 1 grade I not requiring any treatment and 1 grade II requiring an evacuating puncture), 1 cholecystitis grade III requiring a radiological drainage, 1 inhalation grade III causing a pulmonary infection and 2 fever grade I. In the PT group, 2 patients had complications grade III: 1 liver bleeding requiring transfusion of 2 pouches of red blood cells but without specific treatment, and 1 local abscess of $100 \mathrm{~mm}$, with favorable evolution after antibiotic therapy and radiological drainage. There was significantly more cytolysis, evaluated with a blood test at $\mathrm{J}+1$, in the PT+TAC group than in the PT group: the mean AST level was $394 \mathrm{U} / \mathrm{l}$ vs $180 \mathrm{U} / \mathrm{l}$, respectively in the PT+TAC and PT group $(p=0.003)$.

Table 6: 15 complications occurred on 13 patients

\begin{tabular}{|c|c|c|c|c|c|}
\hline & \multicolumn{2}{|c|}{ PT+TAC } & \multicolumn{2}{|c|}{ PT } & \multirow{2}{*}{$\mathrm{p}$-value } \\
\hline & $\mathrm{N}^{+}$ & $\%$ & $\mathrm{~N}^{+}$ & $\%$ & \\
\hline Patients with complications & 8 & 26.7 & 5 & 14.7 & 0.381 \\
\hline Grade & & & & & 0.472 \\
\hline 1 & 5 & 16.8 & 1 & 2.9 & \\
\hline 2 & 3 & 10.1 & 2 & 5.9 & \\
\hline 3 & 2 & 6.7 & 2 & 5.9 & \\
\hline Pain, grade & & & & & 0.747 \\
\hline 1 & 2 & 6.7 & 1 & 2.9 & \\
\hline 2 & 2 & 6.7 & 2 & 5.9 & \\
\hline Bleeding, grade & & & & & 1 \\
\hline 1 & 1 & 3.3 & 0 & 0 & \\
\hline 2 & 1 & 3.3 & 0 & 0 & \\
\hline 3 & 0 & 0 & 1 & 2.9 & \\
\hline Other & & & & & 0.600 \\
\hline Fever grade 1 & 2 & 6.7 & 0 & 0 & \\
\hline Abscess grade 3 & 0 & 0 & 1 & 2.9 & \\
\hline Inhalation grade 3 & 1 & 3.3 & 0 & 0 & \\
\hline Cholecystitis grade 3 & 1 & 3.3 & 0 & 0 & \\
\hline
\end{tabular}

${ }^{\dagger}$ Number of patients 


\section{DISCUSSION}

The association of TAC with PT in a single session for treatment of uninodular HCCs between 30 to $50 \mathrm{~mm}$ and oligonodular HCCs (up to 3 nodules, up to $30 \mathrm{~mm}$ ) seems to catch up to the OS of uninodular HCCs less than $30 \mathrm{~mm}$ treated by PT alone. The 1-, 3-, and 5-year OS in the studied group was $100 \%, 82 \%$ and $48.5 \%$ vs $94.1 \%, 72.7 \%$ and $65.2 \%$ in the PT group $(p=0.88)$. However, these more advanced HCCs recurred faster and required more treatment sessions. The 1-, 3-, and 5-year RFS in the studied group was $59.3 \%, 18.5 \%$ and $5.6 \%$ vs $89.3 \%, 46.4 \%$ and $24.5 \%$ in the PT group ( $p=0.02)$. Fifty-seven percent of patients was treated again one year after the initial treatment versus fifteen percent in the PT group $(p=0.003)$. There was no significant difference in the grade 3 complication rate between the two groups. To our knowledge there are only a few articles evaluating TAC following PT in a single session, and our study is the first to be done on a Western population.

More and more resources are being deployed to widen the range of curative treatments for HCC. Validated curative treatments of HCC include liver transplantation, liver resection and PT by RFA or MWA. The curative radiological treatments under development are multipolar radiofrequency, cryoablation and irreversible electroporation ${ }^{16}$. Looking for innovative technical ways to increase the possibility of curing patients with $\mathrm{HCC}$ of more than $2 \mathrm{~cm}$ of diameter is an important challenge. The association of TAC with RFA for the treatment of intermediate HCC, according to Barcelona Clinic Liver Cancer (BCLC) classification, is known to obtain higher tumor response rates and to improve survival rates than TACE alone ${ }^{17}$. However, most studies perform TAC and PT in two steps, TAC being performed first. The delay between TAC and PT is increasingly being shortened. Okamoto $\mathrm{T}$. et al. presented to the European Hepatology Congress a study into the association of TAC with RFA for intermediate stage HCC where the delay between the two treatments was 1 week $^{18}$. The 1-, 3-, and 5-year OS in the combination treatment group was $97 \%, 70 \%$ and $60 \%$, significantly higher than in TAC alone group. The association of TAC with PT in a single session has been very little studied. Saviano A. et al. compared this association in a single session for treatment of single $\mathrm{HCC} \geq 30 \mathrm{~mm}$ 
(range 30-68 mm) versus liver resection ${ }^{19}$. The 1- and 3-year OS in the TAC+PT group was $89.4 \%$ and $48.2 \%$, which was lower than the OS in our study. However, the HCC was not "naïve-treatment" and many HCC were larger than in our study. There was no significant 3year OS difference between TAC+PT and liver resection. Huyn D. et al. tested the combination TAC+PT in a single time on HCC $<30 \mathrm{~mm}$ infeasible for ultrasound-guided RFA ${ }^{15}$. The 1- and 3-year OS in the TAC+PT group was $100 \%$ and $89 \%$, as for our results ( $100 \%$ and $82 \%)$ although the HCC of our studied group were larger. Finally, Yuan $\mathrm{H}$. et al. compared the combination treatment in a single session for $\mathrm{HCC} \geq 50 \mathrm{~mm}$ versus sequential treatment of TAC and RFA (RFA at 2-4 weeks after TAC). The 1- and 3-year OS of the combination treatment group was $95.8 \%$ and $58.3 \%$ vs $83.3 \%$ and $43.8 \%$ for the sequential group. The difference was significant at 1 year $(p=0.045)$ but not at 3 years $(p=0.153)$. Our work was designed to evaluate the association of TAC with PT in a single session for HCC of intermediate size, a size lying between that of the two above studies. We chose the size criteria according to the "Milan criteria", the benchmark for the selection of patients with HCC for liver transplantation. In the case of a long-anticipated waiting time, patients may be offered treatment to minimize the risk of tumor progression. The association of TAC with PT could be proposed to offer a "bridge" to transplantation. To obtain the purest possible results, we decided to include only treatment-naïve patients with histologically proven HCC, and concordant arteriography.

Early stage HCC patients can be complex to manage. All guidelines recommend a liver transplantation for HCC according to Milan criteria",20-22. There is interest in a "bridging treatment", but the modality of this treatment is not clear. Liver resection, PT or TAC are proposed in turn, favoring resection for large single $\mathrm{HCC}$. But few $\mathrm{HCC}$ are candidates for liver resection due to portal hypertension or major comorbidity. In addition, single HCC between 30 to $50 \mathrm{~mm}$ or oligonodular HCC up to $30 \mathrm{~mm}$ may be treated by TAC, which is a palliative treatment. Most guidelines mention the association of TAC with PT especially for these nodules, reporting that further studies are needed ${ }^{5,20,21}$. Likewise, new ablation techniques are 
mentioned, including multipolar RFA and irreversible electroporation. So, the current challenge is to better treat HCCs that are borderlines between BCLC-A and BCLC-B. Multipolar RFA, cryoablation and irreversible electroporation are performed by only a few expert centers. Potential interest in association of TAC with PT lies in the availability of these techniques in most centers. Our study shows the usefulness of this association for selected HCCs. It should be noted that all of our patients had major comorbidities and/or portal hypertension, so they were rejected for resection. Moreover, only $20 \%$ of patients in the studied group (in Milan criteria) were finally transplanted within the considered period. An increase in this proportion would have probably increased the OS.

The main weakness of our study is the relatively small size of our population, but the results are encouraging and require confirmation in larger cohorts. Moreover, this study is retrospective and done according a non-randomized schema. However, an objective strong primary endpoint, the OS and strict inclusion criteria were obtained. The strengths of our study include the pilot character of the association that has never been studied in a Western population, the long follow-up (up to 5 years) and the rigorous inclusion criteria (histologically proven, naïve-treatment, arteriography concordant).

To conclude, the association of TAC with PT in a single session for treatment of HCC according to Milan criteria is an interesting solution in the current state of knowledge. The OS for this association was equal to unique HCC up to $30 \mathrm{~mm}$ treated by PT alone. However, the occurrence of relapse was more frequent, requiring more intensive monitoring and treatment. These results need to be confirmed in randomized clinical trials. It might be interesting to compare this association with other radiological techniques currently evaluated. Finally, it may be interesting to evaluate the impact of this association on the time to relapse after liver transplantation to validate this treatment as "bridging therapy". 


\section{REFERENCES}

1. Global Cancer Observatory. Estimated number worldwide, GLOBOCAN 2018. http://gco.iarc.fr

2. Survie des personnes atteintes de cancer en France métropolitaine, 1989-2013. Partie 1 - Tumeurs solides, Santé Publique France, INVS

3. Sangiovanni A, Del Ninno E, Fasani $P$ et al. Increased survival of cirrhotic patients with a hepatocellular carcinoma detected during surveillance. Gastroenterology 2004;126:10051014

4. Goutté N, Sogni P, Bendersky N, Barbare JC, Falissard B, Farges O. Geographical variations in incidence, management and survival of hepatocellular carcinoma in a Western country. J Hepatol 2017;66:537-544

5. Blanc JF, Barbare JC, Baumann AS, et al. « Carcinome hépatocellulaire ». Thésaurus National de Cancérologie Digestive, Mars 2019, [En ligne] [http://www.tncd.org]

6. Bruix J, Sherman M ; American Association for the Study of Liver Diseases. Management of hepatocellular carcinoma: an update. Hepatology 2011;53:1020-1022

7. Omata M, Lesmana LA, Tateishi R, et al. Asian Pacific Association for the Study of the Liver consensus recommendations on hepatocellular carcinoma. Hepatol Int 2010;4:439-474

8. Cucchetti A, Piscaglia F, Cescon M, et al. Cost-effectiveness of hepatic resection versus percutaneous radiofrequency ablation for early hepatocellular carcinoma. J Hepatol 2013;59:300-307

9. Nault JC, Sutter O, Nahon P, Ganne-Carrié N, Séror O. Percutaneous treatment of hepatocellular carcinoma: State of the art and innovations. J Hepatol 2018;68:783-797

10. Bruix J, Sherman M; Practice Guidelines Committee, American Association for the Study of Liver Diseases. Management of hepatocellular carcinoma. Hepatology 2005;42:12081236

11. Livraghi T, Goldberg SN, Lazzaroni S, et al. Hepatocellular carcinoma: radio-frequency ablation of medium and large lesions. Radiology 2000;214:761-768

12. Chevallier P, Baudin G, Anty R, et al. Treatment of hepatocellular carcinomas by thermal ablation and hepatic transarterial chemoembolization. Diagn Interv Imaging 2015;96:637-646 
13. Sugimori K, Morimoto M, Shirato K, et al. Radiofrequency ablation in a pig liver model: effect of transcatheter arterial embolization on coagulation diameter and histologic characteristics. Hepatol Res 2002;24:164

14. Yuan H, Liu F, Li X, Guan Y, Wang M. Angio-CT-Guided Transarterial Chemoembolization Immediately in Combination with Radiofrequency Ablation for Large Hepatocellular Carcinoma. Acad Radiol 2019;26:224-231

15. Hyun D, Cho SK, Shin SW, et al. Combined transarterial chemoembolization and radiofrequency ablation for small treatment-naïve hepatocellular carcinoma infeasible for ultrasound-guided radiofrequency ablation: long-term outcomes. Act Radiol 2018;59:773-781

16. Hocquelet A, Aubé C, Rode A et al. Comparison of no-touch multi-bipolar vs. monopolar radiofrequency ablation for small HCC. J Hepatol 2017;66:67-74

17. Yang DJ, Luo KL, Liu $\mathrm{H}$, et al. Meta-analysis of transcatheter arterial chemoembolization plus radiofrequency ablation versus transcatheter arterial chemoembolization alone for hepatocellular carcinoma. Oncotarget 2017;8:2960-2970

18. Okamoto $\mathrm{T}$, Endo $\mathrm{K}$, Takikawa $\mathrm{Y}$ et al. Efficacy of combination therapy with transcatheter arterial chemoembolization and radiofrequency ablation for intermediate-stage hepatocellular carcinoma. ILC 2019, Abstract FRI-493, J Hepatol 2019;70:e615-e616

19. Saviano A, lezzi R, Giuliante $F$ et al. Liver resection versus radiofrequency ablation plus trancatheter arterial chemoembolization in cirrhotic patients with solitary large hepatocellular carcinoma. J Vasc Interv Radiol 2017;28:1512-1519

20. European Association for the Study of the Liver. EASL clinical practice guidelines: management of hepatocellular carcinoma. J Hepatol 2018;69:182-236

21. Heimbach JK, Kulik LM, Finn RS et al. AASLD guidelines for the treatment of hepatocellular carcinoma. Hepatology 2018;67:358-380

22. Vogel A, Cervantes A, Chau I et al. Hepatocellular carcinoma: ESMO Clinical Practice Guidelines for diagnosis, treatment and follow-up. Ann Oncol 2019;30:871-873 


\title{
M Gmail
}

\section{Manuscript submitted - LIVint-19-01157}

\author{
1 message
}

18-Aug-2019

LIVint-19-01157 "Endoarterial treatment in combination with percutaneous thermoablation for medium-sized and oligonodular hepatocellular carcinomas"

Dear Dr. Ouizeman,

The editorial office of Liver International has received your recently submitted manuscript, entitled "Endoarterial treatment in combination with percutaneous thermoablation for medium-sized and oligonodular hepatocellular carcinomas".

You will be informed of the decision as soon as possible. Your manuscript should not be submitted to another journal while under review at Liver International.

Co-authors: Please contact the Editorial Office as soon as possible if you disagree with being listed as a co-author for this manuscript.

Please take note of the assigned number above for the purpose of all future correspondence pertaining to this manuscript.

Thank you for your interest in Liver International.

With kind regards,

Dr. Sarah Bremner Editorial Assistant Liver International 


\section{SERMENT D'HIPPOCRATE}

\section{Ordre National des Médecins Conseil National de l'Ordre}

Au moment d'être admis(e) à exercer la médecine, je promets et je jure d'être fidèle aux lois de l'honneur et de la probité.

Mon premier souci sera de rétablir, de préserver ou de promouvoir la santé dans tous ses éléments, physiques et mentaux, individuels et sociaux.

Je respecterai toutes les personnes, leur autonomie et leur volonté, sans aucune discrimination selon leur état ou leurs convictions. J'interviendrai pour les protéger si elles sont affaiblies, vulnérables ou menacées dans leur intégrité ou leur dignité. Même sous la contrainte, je ne ferai pas usage de mes connaissances contre les lois de l'humanité.

J'informerai les patients des décisions envisagées, de leurs raisons et de leurs conséquences. Je ne tromperai jamais leur confiance et n'exploiterai pas le pouvoir hérité des circonstances pour forcer les consciences.

Je donnerai mes soins à l'indigent et à quiconque me les demandera. Je ne me laisserai pas influencer par la soif du gain ou la recherche de la gloire.

Admis(e) dans l'intimité des personnes, je tairai les secrets qui me seront confiés. Reçu(e) à l'intérieur des maisons, je respecterai les secrets des foyers et ma conduite ne servira pas à corrompre les mœurs.

Je ferai tout pour soulager les souffrances. Je ne prolongerai pas abusivement les agonies. Je ne provoquerai jamais la mort délibérément.

Je préserverai l'indépendance nécessaire à l'accomplissement de ma mission. Je n'entreprendrai rien qui dépasse mes compétences. Je les entretiendrai et les perfectionnerai pour assurer au mieux les services qui me seront demandés.

J'apporterai mon aide à mes confrères ainsi qu'à leurs familles dans l'adversité.

Que les hommes et mes confrères m'accordent leur estime si je suis fidèle à mes promesses ; que je sois déshonoré(e) et méprisé(e) si j’y manque. 